\title{
May 2014 Arizona Thoracic Society Notes
}

The May 2014 Arizona Thoracic Society meeting was held on Wednesday, 5/28/2014 at Scottsdale Shea Hospital beginning at 6:30 PM. There were 13 in attendance representing the pulmonary, critical care, sleep and radiology communities.

A discussion was held regarding the Arizona Thoracic Society relationship with the American Lung Association. Several members volunteered to talk to the lung association regarding common ground to strengthen the relationship.

The wine tasting with the California, New Mexico and Colorado Thoracic Societies at the American Thoracic Society International Meeting was a big success. There were about 55 at the meeting. The tasting will probably be held again next year.

At the ATS meeting data was presented that pirfenidone was effective in reducing the progression of idiopathic pulmonary fibrosis (IPF). The data was published in the New England Journal of Medicine on 8/29/14 (1). Lewis Wesselius is one of the investigators enrolling patients in a phase 3 trial while InterMune reapplies to the FDA for approval of pirfenidone in IPF.

Two cases were presented:

1. Lewis Wesselius from the Mayo Clinic Arizona presented a 53 year old woman with a chronic, nonproductive accompanied by malaise and a modest weight loss. She was treated for asthma without improvement. She was a nonsmoker and had a SpO2 of $98 \%$ on room air. Her lungs were clear to auscultation. Routine laboratory evaluation was unremarkable and exhaled nitric oxide was normal. Thoracic CT scan showed a subtle broncholitis. She was empirically treated for gastroesophageal reflux disease (GERD) without improvement. Bronchoalveolar lavage was performed and showed Nocardia asteroides. She had no evidence of immunocompromise. She was treated with sulfamethoxazole and trimethoprim which produced a rash and then minocycline for 4 months. Her cough resolved. However, when the minocycline was stopped her cough returned. She is currently receiving an additional course of minocycline planned for 6 months.

2. Suresh Uppalapu presented a 58 year old fireman with a complaint of dyspnea on exertion. He has a history of obstructive sleep apnea and lives at an elevation of 7000 feet. The patient had significant desaturation with exercise. Chest x-ray showed borderline cardiomegaly but was otherwise normal. Thoracic CT scan showed pulmonary artery enlargement and borderline right ventricular (RV) enlargement. Ultrasound of the hear showed an enlarged RV but it was difficult to measure PA pressure. Right-sided heart catherization showed a mean pulmonary artery pressure of $35 \mathrm{~cm} \mathrm{H} 2 \mathrm{O}$ with a 
step up in the oxygen saturation at the right atrium. Transesophageal echocardiogram (TEE) showed a patent foramen ovale (PFO). Insertion of a balloon stopped the right to left shunting but resulted in a significant increase in the pulmonary artery pressure. He was referred for percutaneous closure of the PFO along with treatment of his pulmonary artery hypertension.

There being no further business the meeting was adjourned about 8:15 PM. The June meeting is scheduled for Tucson. There will be no meeting in July. The next meeting in Phoenix will be a case presentation conference on August 27, 6:30 PM at Scottsdale Shea Hospital.

Richard A. Robbins, MD

\section{Reference}

1. King TE Jr, Bradford WZ, Castro-Bernardini S, et al. A phase 3 trial of pirfenidone in patients with idiopathic pulmonary fibrosis. N Engl J Med 2014;370:2083-92. [CrossRef] [PubMed] 